\title{
Automatic Design of Binary W-Operators Using Artificial Feed-Forward Neural Networks Based on the Weighted Mean Square Error Cost Function
}

\author{
Marco Benalcázar ${ }^{1,2,3}$, Marcel Brun ${ }^{1}$, Virginia Ballarin ${ }^{1}$, Isabel Passoni ${ }^{4}$, \\ Gustavo Meschino ${ }^{4}$, and Lucía Dai Pra ${ }^{4}$ \\ ${ }^{1}$ Grupo de Procesamiento de Imágenes, Universidad Nacional de Mar del Plata, Argentina \\ ${ }^{2}$ Consejo Nacional de Investigaciones Científicas y Técnicas, Argentina \\ ${ }^{3}$ Secretaría Nacional de Educación Superior Ciencia, Tecnología e Innovación, Ecuador \\ marco_benalcazar@hotmail.com \\ http: //www3.fi.mdp. edu.ar/imagenes \\ ${ }^{4}$ Laboratorio de Bioingeniería, Universidad Nacional de Mar del Plata, Argentina
}

\begin{abstract}
One of the main issues concerning automatic design of W-operators is the one of generalization. Considering the designing of W-operators as a particular case of designing a pattern recognition system, in this work we propose a new approach for the automatic design of binary W-operators. This approach consists on a functional representation of the conditional probabilities for the whole set of patterns viewed by a given window, instead the values of the characteristic function. The estimation of its parameters is achieved by means of a nonlinear regression performed by an artificial feed-forward neural network based on a weighted mean square error cost function. Experimental results show that, for the applications presented in this work, the proposed approach leads to better results than one of the best existing methods of generalization within the family of $\mathrm{W}$-operators, like is the case of pyramidal multiresolution.
\end{abstract}

Keywords: W-operators, pattern recognition, artificial neural network, nonlinear regression, weighted mean square error, pyramidal multiresolution.

\section{Introduction}

The W-operators are a class of nonlinear operators, within the domain of Computational Mathematical Morphology [1,2]. The designing process of these operators is based on the estimation of joint probabilities [3], or conditional probabilities [4], for the patterns viewed by a given window. The estimation is made from training examples, formed by pairs of observed and ideal images. Observed images represent the problem to be solved and ideal images correspond to the desired output. Using these probabilities, W-operators are designed and characterized by characteristic functions that minimize some given cost function or error measure.

One of the main issues that appear in the automatic design of W-operators is the one of generalization. It occurs when certain patterns are not found during training. 
Some approaches have been proposed to circumvent this problem such as: automatic programming of binary morphological machines, pyramidal multiresolution [1], decision trees, genetic algorithms, adaptive algorithms, incremental splitting intervals (ISI), and multilevel training [3].

In this paper we propose a new approach for generalization in the design of W-operators: instead of generalizing the characteristic function, computed from the estimation of the conditional probabilities, we apply artificial neural networks to approximate the conditional probabilities, and then deduce the characteristic function from this approximation. We chose artificial neural networks because of their ability of generalization and because they may generate complex nonlinear boundaries [6]. Finally, the network learning is based on the weighted mean square error (WMSE) cost function [7].

Following this introduction, Section 2 recalls the formulation of the statistical design of binary W-operators. Section 3 describes the proposed approach. In Section 4, we present two application examples, and finally, in Section 5, we summarize this work and outline future possible developments.

\section{W-Operators}

Let consider a binary image as a function $H: \boldsymbol{E} \rightarrow\{0,1\}$, where $\boldsymbol{E}$ is a rectangular subset of $\mathbf{Z}^{2}$. The set of all possible images from $\boldsymbol{E}$ to $\{0,1\}$ will be denoted by $\{0,1\}^{\mathbf{E}}$. A point $t=(x, y)$ of this image, or pixel, is a coordinate in $\boldsymbol{E}$, where $x$ and $y$ are integer numbers. Finally, a window $W=\left\{w_{1}, w_{2}, \ldots, w_{n}\right\}$ is another small subset of $\mathbf{Z}^{2}$, usually including the origin of coordinates of $\boldsymbol{E}:(0,0) \in W$, with $w_{k} \in \mathbf{Z}^{2}$, denoting the size of the window $W$ by $n=|W|$.

Given a binary image $H$, an arbitrary point $t$ of this image, and a window $W$. A window configuration, or observation $\mathbf{u}_{H(t)}=\left(u_{1}, \ldots, u_{n}\right)$, is a vector composed of $n$ binary values: $u_{k} \in\{0,1\}$, where each value is defined by $u_{k}=H\left(t+w_{k}\right)$; i.e., by the image value at the point $w_{k}+t$ of the window $W_{t}$, where $W_{t}$ denotes the translation of $W$ at the point $t: W_{t}=\{w+t: w \in W\}$. Thus, $\mathbf{u}_{H(t)}$ can be expressed as follows:

$$
\mathbf{u}_{H(t)}=\left(H\left(w_{1}+t\right), H\left(w_{2}+t\right), \ldots, H\left(w_{n}+t\right)\right)
$$

Since $\mathbf{u}_{H(t)} \in\{0,1\}^{n}$, the number of possible observations for a given window $W$ is $2^{n}$. On the other hand, a W-operator, $\boldsymbol{\Psi}$, is a function of the form $\Psi:\{0,1\}^{\mathbf{E}} \rightarrow\{0,1\}^{\mathbf{E}}$ that, applied to the binary image $H$, produces other binary image $G=\mathbf{\Psi}(H)$. Moreover, W-operators are characterized by binary functions, called characteristic functions, whose form is $\psi:\{0,1\}^{n} \rightarrow\{0,1\}$, such that, for each point $t$ $\in \boldsymbol{E}$ :

$$
G(t)=\boldsymbol{\Psi}(H)(t)=\psi\left(H\left(w_{1}+t\right), H\left(w_{2}+t\right), \ldots, H\left(w_{n}+t\right)\right)=\psi\left(\mathbf{u}_{H(t)}\right)
$$


Finally, W-operators fulfill two important properties: translation invariance, which means that $\boldsymbol{\Psi}\left(H_{t}\right)=\boldsymbol{\Psi}(H)_{t}$; and local definition within $W$, which implies that $\boldsymbol{\Psi}(H)(t)=\boldsymbol{\Psi}\left(H \cap W_{t}\right)(t)$. The last property indicates that knowing only the values of $H$ in the neighborhood of $W_{t}$, we can determine the value of $\boldsymbol{\Psi}(H)(t)[1,2]$.

\subsection{Statistical Design of Binary W-Operators}

To perform the statistical design of $\mathrm{W}$-operators, we model the images to be processed (i.e., observed images) and their desired images (i.e., ideal images) as realizations of two stationary and stochastic processes $\mathbf{O}$ and $\mathbf{I}$, respectively [1,2]. In this context, the goal of the statistical design is to find a W-operator $\Psi$ such that, given realizations $(I, O)$ from the processes $(\mathbf{I}, \mathbf{O}), \Psi(O)$ must be as close as possible to $I$. Using the mean square error (MSE) as the closeness measure between $I$ and $\Psi(O)$, we can establish, at the origin of coordinates $(0,0)=0$, the following relationship as the cost function for the statistical design of binary $\mathrm{W}$-operators:

$$
M S E=\sum_{\{\mathbf{u}: \mathbf{u} \in O \cap W(0)\}} \operatorname{Pr}(I(0) \neq \psi(\mathbf{u}) \mid \mathbf{u}) \operatorname{Pr}(\mathbf{u})
$$

In the above equation, $\operatorname{Pr}(I(0) \neq \psi(\mathbf{u}) \mid \mathbf{u})$ is the conditional probability, given the observation $\mathbf{u} \in O \cap W_{(0)}$, that $I(0)$ is different from the result of applying the characteristic function $\psi$ to the observation $\mathbf{u}$; while $\operatorname{Pr}(\mathbf{u})$ represents the marginal probability, or frequency, that $\mathbf{u}$ occurs in the image $O \cap W_{(0)}$. Therefore, the optimal binary Woperator $\Psi_{\text {opt }}$, represented by its characteristic function $\psi_{o p t}$, minimizes equation (3). Formally, $\psi_{\text {opt }}$ is defined by the following expression:

$$
\psi_{\text {opt }}(\mathbf{u})=\left\{\begin{array}{lll}
1 & \text { if } & \operatorname{Pr}(I(0)=1 \mid \mathbf{u}) \geq \operatorname{Pr}(I(0)=0 \mid \mathbf{u}) \\
0 & \text { if } & \operatorname{Pr}(I(0)=1 \mid \mathbf{u})<\operatorname{Pr}(I(0)=0 \mid \mathbf{u})
\end{array}\right.
$$

Due to the fact that in practice the conditional probabilities $\operatorname{Pr}(I(0)=1 \mid \mathbf{u})$ and $\operatorname{Pr}(I(0)=0 \mid \mathbf{u})$ are not known, we may estimate their values using pairs of training images, and then determine the value of the characteristic function $\psi(\mathbf{u})$ by applying equation (4) [2]. On the other hand, in order to use equation (4), the values of $\hat{\operatorname{Pr}}(I(0)=1 \mid \mathbf{u})$ and $\hat{\operatorname{Pr}}(I(0)=0 \mid \mathbf{u})$, for a given observation $\mathbf{u}$, need to be known. Since good estimation of probabilities in practice is not always possible because the amount of training data is limited, and also considering that the number of possible window configurations grows exponentially with the size of the window $W$, we propose to use artificial neural networks to approximate the conditional probabilities for the whole set of window configurations $\left\{\mathbf{u}_{i}: i=1, \ldots, 2^{n} ; \quad n=|W|\right\}$, depending on the available training data, instead of generalizing the class labels of the window configurations. 


\section{Generalizing Conditional Probabilities Using Artificial Feed- Forward Neural Networks}

In this section, we describe the proposed generalization for the automatic design of binary $\mathrm{W}$-operators. This approach consists of finding two functions that, for any window configuration $\mathbf{u}$, approximate the estimated values of the conditional probabilities $\operatorname{Pr}(I(0)=1 \mid \mathbf{u})$ and $\operatorname{Pr}(I(0)=0 \mid \mathbf{u})$. In order to achieve such generalization, we use neural networks, a supervised learning tool from Machine Learning, that are widely used to solve classification and regression problems [6-8]. In our case, the neural networks are used to estimate the parameters of a nonlinear regression.

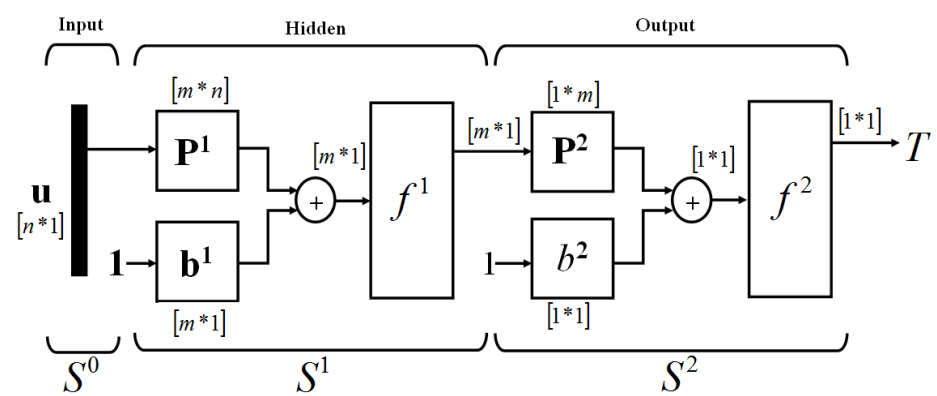

Fig. 1. Three layer artificial feed-forward neural network architecture

Based on the fact that an artificial feed-forward neural network of three layers is capable of approximating any Borel measurable function [9], for the network architecture shown in Fig. 1 (using the representation from [8]), where the layer $S^{1}$ is composed of $m$ neurons, with sigmoid transfer functions $f^{1}$, and the single one neuron of the layer $S^{2}$ has a linear transfer function $f^{2}(z)=z$, the response $T$, for an input vector $\mathbf{u}=\left(u_{1}, \ldots, u_{n}\right)$, is given by the following equation:

$$
T(\mathbf{u})=f^{2}\left(\mathbf{P}^{2} f^{1}\left(\mathbf{P}^{1} \mathbf{u}+\mathbf{b}^{1}\right)+b^{2}\right)=\mathbf{P}^{2} f^{1}\left(\mathbf{P}^{1} \mathbf{u}+\mathbf{b}^{1}\right)+b^{2},
$$

where, $\mathbf{P}^{1}$ and $\mathbf{P}^{2}$ represent the weight matrices of $S^{1}$ and $S^{2}$, respectively, whereas $\mathbf{b}^{1}$ represents the bias matrix of $S^{1}$, and $b^{2}$ is the bias of $S^{2}$.

Moreover, starting from the second axiom of probability, for any observation $\mathbf{u}$, the sum of conditional probabilities must satisfy: $\operatorname{Pr}(I(0)=1 \mid \mathbf{u})+\operatorname{Pr}(I(0)=0 \mid \mathbf{u})=1$. Adding $2 \delta$ to both sides of this expression, where $\delta \in \mathfrak{R}^{+}$is an infinitesimal number (e.g. the distance between 1 and the closest double-precision number: $\left.2^{-52}\right)$, and conveniently grouping terms, we obtain: $(\operatorname{Pr}(I(0)=1 \mid \mathbf{u})+\delta)+(\operatorname{Pr}(I(0)=0 \mid \mathbf{u})+\delta)=1+2 \delta$. Then, computing the natural logarithm of the ratio between the two terms on the left side of this equation, we have: $\phi(\mathbf{u})=\operatorname{Ln}\left(\frac{\operatorname{Pr}(I(0)=1 \mid \mathbf{u})+\delta}{\operatorname{Pr}(I(0)=0 \mid \mathbf{u})+\delta}\right)=\operatorname{Ln}\left(\frac{\operatorname{Pr}(I(0)=1 \mid \mathbf{u})+\delta}{1-\operatorname{Pr}(I(0)=1 \mid \mathbf{u})+\delta}\right)$. In this 
equation, if $\delta=0$, the resulting expression resembles that one used for defining a logistic regression. Also, It is worth noticing that $\delta$ allows us to represent, with finite values, the cases when $\operatorname{Pr}(I(0)=1 \mid \mathbf{u})=0$ and $\operatorname{Pr}(I(0)=0 \mid \mathbf{u})=1$, or $\operatorname{Pr}(I(0)=1 \mid \mathbf{u})=1$ and $\operatorname{Pr}(I(0)=0 \mid \mathbf{u})=0$. Furthermore, approximating the right side of equation (5) with the right side of the last expression, we obtain the following relationship that defines a nonlinear regression:

$$
\operatorname{Ln}\left(\frac{\operatorname{Pr}(I(0)=1 \mid \mathbf{u})+\delta}{\operatorname{Pr}(I(0)=0 \mid \mathbf{u})+\delta}\right)=\operatorname{Ln}\left(\frac{\operatorname{Pr}(I(0)=1 \mid \mathbf{u})+\delta}{1-\operatorname{Pr}(I(0)=1 \mid \mathbf{u})+\delta}\right)=\mathbf{P}^{2} f^{1}\left(\mathbf{P}^{1} \mathbf{u}+\mathbf{b}^{1}\right)+b^{2}
$$

From the above equation, it follows that the function that generalizes the conditional probability $\operatorname{Pr}(I(0)=1 \mid \mathbf{u})$, for the window configuration $\mathbf{u}$, has the following form:

$$
\operatorname{Pr}(I(0)=1 \mid \mathbf{u})=\frac{\exp \left(\mathbf{P}^{2} f^{1}\left(\mathbf{P}^{1} \mathbf{u}+\mathbf{b}^{1}\right)+b^{2}\right)(1+\delta)-\delta}{1+\exp \left(\mathbf{P}^{2} f^{1}\left(\mathbf{P}^{1} \mathbf{u}+\mathbf{b}^{1}\right)+b^{2}\right)}
$$

while $\operatorname{Pr}(I(0)=0 \mid \mathbf{u})$ is generalized by the following expression:

$$
\operatorname{Pr}(I(0)=0 \mid \mathbf{u})=\frac{\left(1-\exp \left(\mathbf{P}^{2} f^{1}\left(\mathbf{P}^{1} \mathbf{u}+\mathbf{b}^{1}\right)+b^{2}\right)\right) \delta+1}{1+\exp \left(\mathbf{P}^{2} f^{1}\left(\mathbf{P}^{1} \mathbf{u}+\mathbf{b}^{1}\right)+b^{2}\right)}
$$

Therefore, given the conditional probabilities $\operatorname{Pr}\left(I(0)=1 \mid \mathbf{u}_{j}\right)$ and $\operatorname{Pr}\left(I(0)=0 \mid \mathbf{u}_{j}\right)$, estimated in a first stage of the proposed designing process by means of the relative frequencies $\frac{\text { freq }\left(I=1 \mid \mathbf{u}_{j}\right)}{\text { freq }\left(I=0 \mid \mathbf{u}_{j}\right)+\text { freq }\left(I=1 \mid \mathbf{u}_{j}\right)}$ and $\frac{\text { freq }\left(I=0 \mid \mathbf{u}_{j}\right)}{\text { freq }\left(I=0 \mid \mathbf{u}_{j}\right)+\text { freq }\left(I=1 \mid \mathbf{u}_{j}\right)}$, respectively, with $j=1, \ldots, l$, where $l$ is the total number of different configurations collected by shifting the window in the training images, the goal of a second stage is to estimate the weights $\mathbf{P}^{1}$ and $\mathbf{P}^{2}$, and the bias $\mathbf{b}^{1}$ and $b^{2}$ for an artificial feed-forward neural network, using equation (6). Moreover, the network training will be based on the WMSE cost function, allowing us to use the marginal probability, or frequency, $\operatorname{Pr}\left(\mathbf{u}_{j}\right)$ of each observation $\mathbf{u}_{j}$ collected in the first stage.

\subsection{Training an Artificial Feed-Forward Neural Network Based on the Weighted Mean Square Error Cost Function}

The use of the WMSE function $M S E_{W}$, defined by equation (9), as the cost function for training an artificial neural network has two important advantages compared with the MSE: 1) the noise-contaminated observations have less influence on the cost function and 2) if there are no noisy observations, the network can avoid over-fitting [7].

$$
M S E_{W}=\frac{1}{l} \sum_{j=1}^{l}\left(\left(T\left(\mathbf{u}_{j}\right)-\phi\left(\mathbf{u}_{j}\right)\right)^{2} \frac{\operatorname{Pr}\left(\mathbf{u}_{j}\right)}{\max \left(\left\{\operatorname{Pr}\left(\mathbf{u}_{1}\right), \ldots, \operatorname{Pr}\left(\mathbf{u}_{l}\right)\right\}\right)}\right)
$$


In equation (9), $T\left(\mathbf{u}_{j}\right)$ is the network output, $\phi\left(\mathbf{u}_{j}\right)$ is the expected output and $\operatorname{Pr}\left(\mathbf{u}_{j}\right)$ is the marginal probability with that the observation $\mathbf{u}_{j}$ appears in the training images.

As in the case of $\operatorname{Pr}(I(0)=1 \mid \mathbf{u})$ and $\operatorname{Pr}(I(0)=0 \mid \mathbf{u})$ that are not known, $\operatorname{Pr}(\mathbf{u})$ is also unknown, therefore, in equation (9) we must work with its estimate $\hat{\operatorname{Pr}}(\mathbf{u})$. Finally, to complete the definition of the network architecture to perform the nonlinear regression, we determined heuristically based on trial and error, that the best results were obtained when the number of neurons of $S^{1}$ is $m=4 \sqrt{n}$, where $n=|W|$, and the transfer functions for the neurons of this layer are $f^{1}=\operatorname{tansig}(\mathrm{z})=\frac{2}{1+\exp (-2 z)}-1$. The method to be employed for the network training is the Levenberg-Marquardt method, using equation (9) as the cost function to be minimized.

\section{Experimental Results}

In this section, we present two applications of the proposed approach. The goal of the first experiment is to filter the noise in images of retinal angiographies. The angiographies were segmented using an algorithm based on Fuzzy Mathematical Morphology (FMM) [10]. The dataset of this experiment is formed by 4 pairs of observed and ideal images of $565 \times 584$ pixels. Observed images are the segmented ones, and their associated ideal images are taken from the DRIVE database [11].

The goal of the second experiment is to detect edges in objects contained in noisy images. For this case, the dataset is composed of 4 noiseless images of the following sizes: 350x156, 300x270, 637x563 and 401x393 pixels. Observed images are obtained adding synthetic salt-pepper noise, with a density of 0.1 , to images from the dataset. Each ideal image (i.e., image with the edges) is obtained by applying the morphological gradient $H-\varepsilon_{\mathbf{B}}(H)$ to each noiseless image $H$, where $\varepsilon_{\mathbf{B}}(H)$ denotes the erosion of the image $H$ by the structuring element $B$. For the current experiment, we use a square structuring element of size $3 \times 3$ pixels (connectivity 8 border).

Table 1 summarizes the results from noise filtering in images of retinal angiographies, both before and after the application of $\mathrm{W}$-operators. According to these values, the proposed approach produces a substantial reduction of noise in the segmentation of angiographies, compared with pyramidal multiresolution, which can be corroborated by the error rate (ER) and the false positive rate (FPR) values. In the case of the false negative rate (FNR), multiresolution has a slight advantage over Woperators designed using neural networks.

Table 1. Results of noise filtering in images of retinal angiographies

\begin{tabular}{lccc}
\hline & ER [\%] & FPR [\%] & FNR [\%] \\
\hline Original values (FMM algorithm) & 4.53 & 1.89 & 32.78 \\
Nonlinear regression with neural network & 3.94 & 1.50 & 28.86 \\
Pyramidal multiresolution & 4.15 & 1.85 & 27.66 \\
\hline
\end{tabular}


Fig. 2 shows an example of the generalization with neural networks (Fig. 2-c) and pyramidal multiresolution (Fig. 2-d) to the sample image of Fig. 2-b. The image shown in Fig. 2-b is the result of applying FMM segmentation to image of Fig. 2-a.

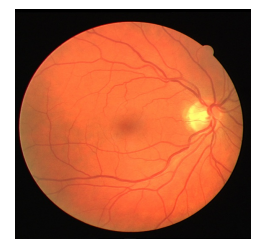

(a)

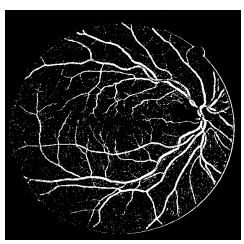

(b)

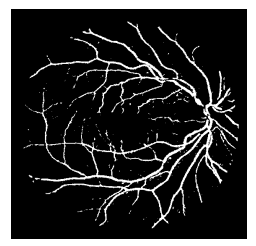

(c)

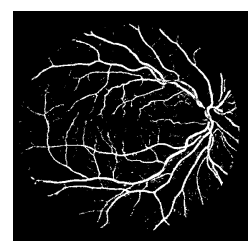

(d)

Fig. 2. (a) Original image, (b) segmented image with FMM; noise filtering by a W-operator based on: (c) neural network and (d) pyramidal multiresolution

Table 2 summarizes the results obtained applying W-operators for edge detection in noisy images. According to the ER, FPR and FNR values, the proposed approach seems more robust to noise compared with pyramidal multiresolution and morphological gradient. Moreover, it is worth noticing that the automatic methods to design Woperators lead to better results than the heuristic method.

Table 2. Results of edge detection in noisy images

\begin{tabular}{lccl}
\hline & ER [\%] & FPR [\%] & FNR [\%] \\
\hline Nonlinear regression with neural network & 1.55 & 0.46 & 17.49 \\
Pyramidal multiresolution & 2.17 & 0.60 & 24.33 \\
Morphological gradient & 22.36 & 18.37 & 94.83 \\
\hline
\end{tabular}

Fig. 3 shows an example of the result of applying the generalization with neural networks (Fig. 3-b), pyramidal multiresolution (Fig. 3-c) and morphological gradient (Fig. 3-d) to the sample image of Fig. 3-a.

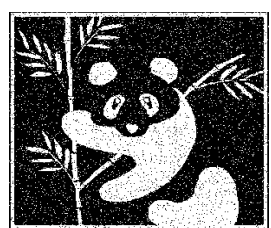

(a)

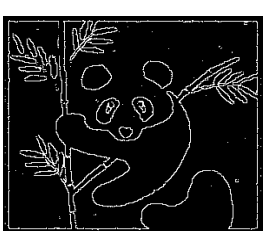

(b)

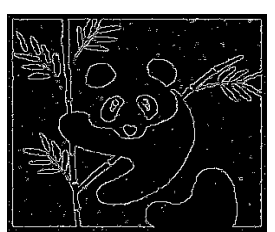

(c)

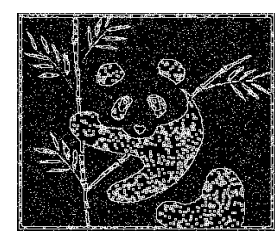

(d)

Fig. 3. (a) Salt-pepper noisy image; edge detection using W-operators based on: (b) neural network, (c) pyramidal multiresolution, and (d) morphological gradient

\section{$5 \quad$ Concluding Remarks}

In this paper we have proposed a new approach for the automatic design of binary Woperators. The idea behind this work is to consider the design of $\mathrm{W}$-operators as a 
particular case of designing a pattern recognition system. Thus, the method consist in the estimation of the parameters of two functions that generalize the conditional probabilities for the whole set of window configurations. The estimation is achieved by nonlinear regression performed by an artificial feed-forward neural network of three layers, whose training is based on the minimization of the WMSE cost function.

This approach allows us to represent W-operators by the parameters of the trained neural network, avoiding the storage of the large amount of data needed to represent characteristic functions, as in techniques like ISI or pyramidal multiresolution.

The experimental results show that, for the examples presented in this work, the proposed approach allows us to achieve better results than one of the best existing methods for the generalization of characteristic functions of $\mathrm{W}$-operators, like is the case of the pyramidal multiresolution.

Further work should include the extension of the proposed method for the automatic design of $\mathrm{W}$-operators to process gray-scale and color images.

\section{References}

1. Hirata Jr., R., Brun, M., Barrera, J., Dougherty, E.R.: Aperture filters: theory, application, and multiresolution analysis. In: Advances in Nonlinear Signal and Image Processing, New York (2006)

2. Barrera, J., Banon, G., Dougherty, E.R.: Automatic design of morphological operators. Space Structure and Randomness. In: Bickel, P., Diggle, P., Fienberg, S., Gather, U., Olkin, I. (eds.) LNS, vol. 183, pp. 257-278. Springer (2005)

3. Santos, C., Hirata, N.S., Hirata, R.: An Information Theory framework for two-stage binary image operator design. Pattern Recognition 31(4), 297-306 (2010)

4. Benalcázar, M., Padín, J., Bouchet, A., Brun, M., Ballarin, V.: Diseño Auto-mático de Operadores Morfológicos Aplicado a la Segmentación de Angiograf-1́as Retinales. In: JAIIO 2011, Argentina, pp. 137-147 (2011)

5. Hirata Jr., R., Dougherty, E.R., Barrera, J.: Aperture Filters. Signal Processing 80(4), 697$721(2000)$

6. Egmont-Petersen, M., Ridder, D., Handels, H.: Image processing with neural network - a review. Pattern Recognition 35 (2002)

7. Sai, Y., Jinxia, R., Zhongxia, L.: Learning of Neural Networks Based on Weighted Mean Squares Error Function. In: IEEE - Computational Intelligence and Design, pp. 241-244 (2009)

8. Hagan, M., Demuth, H., Beale, M.: Neural Network Design, Boston (1996)

9. Hornik, K., Stinchcombe, M., White, H.: Multilayer feed forward networks are universal approximators. Neural Networks 2(5), 359-366 (1989)

10. Bouchet, A., Brun, M., Ballarin, V.: Morfología Matemática Difusa aplicada a la segmentación de angiografías retinales. Revista Argentina de Bioingeniería 16(1), 7-10 (2001)

11. Staal, J., Abramoff, M., Niemeijer, M., Viergever, M., Van Ginneken, B.: Ridge based vessel segmentation in color images of the retina. IEEE Transactions on Medical Imaging 23(4), 501-509 (2004) 